INPLASY

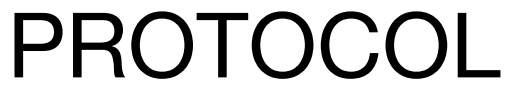

To cite: Ribeiro et al.

Methodological

characteristics, physiological

and physical effects and future

directions for concurrent

training in soccer: A scoping

review. Inplasy protocol

2020110132. doi:

10.37766/inplasy2020.11.0132

Received: 28 November 2020

Published: 28 November 2020

Corresponding author:

Filipe Manuel Clemente

filipe.clemente5@gmail.com

Author Affiliation:

Escola Superior Desporto e Lazer, Instituto Politécnico de Viana do

Castelo, Viana do Castelo,

Portugal

Support: None.

Review Stage at time of this submission: Piloting of the study selection process.

Conflicts of interest:

The authors declare that they have no conflicts of interest relevant to the content of this review.

\section{Methodological characteristics, physiological and physical effects and future directions for concurrent training in soccer: A scoping review}

Ribeiro, J1; Camões, M2; Afonso, J3; Sarmento, H4; Lima, R5; Clemente, $\mathrm{FM}^{6}$.

Review question / Objective: This scoping review was conducted to: (1) characterize the main elements of CT studies (e.g., training protocols) conducted in soccer; (2) summarize the main physiological and physical effects of CT on soccer players; and (3) provide future directions for research.

Condition being studied: CT interventions in soccer players of any age or sex without injury or illness reported.

Information sources: Electronic databases (Web of Science, Scopus, SPORTDiscus and PubMed) were searched for relevant publications prior to the end of December of 2020. Keywords and synonyms were entered in various combinations (i.e., "Soccer" OR "Football") AND ("concurrent training" OR "combined training" OR "resistance training" OR "aerobic training" OR "endurance training" OR "strength training" OR "plyometrics" OR "calisthenics" OR "aerobic" OR "mobility" OR "flexibility" OR "balance" OR "propriocepti"). Additionally, the reference lists of the studies retrieved were manually searched to identify potentially eligible studies not captured by the electronic searches. Finally, an external expert has been contacted in order to verify the final list of references included in this scoping review in order to understand if there was any study that was not detected through our research.

INPLASY registration number: This protocol was registered with the International Platform of Registered Systematic Review and Meta-Analysis Protocols (INPLASY) on 28 November 2020 and was last updated on 01 December 2020 (registration number INPLASY2020110132).

\section{INTRODUCTION}

Review question / Objective: This scoping review was conducted to: (1) characterize the main elements of CT studies (e.g., training protocols) conducted in soccer; (2) summarize the main physiological and physical effects of CT on soccer players; 
and (3) provide future directions for research.

Rationale: Despite some reviews have been publishing about the effects of CT in performance outcomes and physiological changes (Methenitis, 2018; Wilson et al., 2012), there is a lack of systematization in the specific case of soccer. Additionaly, despite some reviews about methodological use of CT in in sports (Baar, 2014; Balsalobre-Fernández, SantosConcejero, \& Grivas, 2016; Izquierdo \& García, 2011), there is a lack of characterization of the training protocols in soccer. For the above-mentioned reasons, there is a need for a scoping review that may help to characterize the experimental CT protocols in soccer players and a general overview of the physiological and physical effects on the players. A scoping review may help to achieve an overview about the possibilities for application of CT on soccer, as well as help to define future research and intervention directions. Therefore, the aim of this scoping review was threefold: (1) characterize the main elements of CT studies (e.g., training protocols) conducted in soccer; (2) summarize the main physiological and physical effects of CT on soccer players; and (3) provide future directions for research.

Condition being studied: CT interventions in soccer players of any age or sex without injury or illness reported.

\section{METHODS}

Search strategy: Keywords and synonyms were entered in various combinations (i.e., "Soccer" OR "Football") AND ("concurrent training" OR "combined training" OR "cross training").

Participant or population: Soccer players of any age or sex without injury or illness reported.

Intervention: Intervention is CT using strength (e.g., including any type of structured strength training, namely, resistance training, plyometrics, calisthenics) and endurance training.
Comparator: Compared with control (passive control with just regular fieldbased training and no other additional program reported) or other intervention group (active control with field-based training and other intervention protocol not consisting in CT, e.g., mobility, agility, or even single interventions of strength or endurance training).

Study designs to be included: The study designs must be experimental (randomized or not randomized) or cohorts.

Eligibility criteria: Inclusion criteria: (i) Population: Soccer players of any age or sex without injury or illness reported; (ii) intervention: Intervention is CT using strength (e.g., including any type of structured strength training, namely, resistance training, plyometrics, calisthenics) and endurance training; (iii) comparator: Compared with control (passive control with just regular fieldbased training and no other additional program reported) or other intervention group (active control with field-based training and other intervention protocol not consisting in CT, e.g., mobility, agility, or even single interventions of strength or endurance training); (iv) outcome: Must have at least one pre-post acute and/or a chronic outcome (acute response: immediate response of a physical or physiological variable in response to the exercise; chronic response: adaptations promoted by the training intervention, consisting in permanent changes in physical or physiological variables) related to physiological (e.g., heart rate responses, blood lactate concentrations, oxygen uptake, rate of perceived exertion) and physical (e.g., strength and power, speed, change-of-direction, aerobic capacity) measures; (v) study design: The study designs must be experimental (randomized or not randomized) or cohorts; and (vi) Only original and full-text studies written in English.

Information sources: Electronic databases (Web of Science, Scopus, SPORTDiscus and PubMed) were searched for relevant publications prior to the end of December 
of 2020. Keywords and synonyms were entered in various combinations (i.e., "Soccer" OR "Football") AND ("concurrent training" OR "combined training" OR "resistance training" OR "aerobic training" OR "endurance training" OR "strength training" OR "plyometrics" OR "calisthenics" OR "aerobic" OR "mobility" OR "flexibility" OR "balance" OR "propriocepti"*"). Additionally, the reference lists of the studies retrieved were manually searched to identify potentially eligible studies not captured by the electronic searches. Finally, an external expert has been contacted in order to verify the final list of references included in this scoping review in order to understand if there was any study that was not detected through our research.

Main outcome(s): Identification of the effects (acute and/or chronic), dimension of analysis (internal load [the measure of biological response to a given physical demand imposed by the exercise (Impellizzeri, Marcora, \& Coutts, 2019)] or biological responses in exercise; external load [the measure of physical demand or neuro-mechanical load imposed by the exercise (Impellizzeri et al., 2019)] or physical demands in exercise; recovery/ fatigue/readiness; psychological; fitness variations), outcomes explored, and main findings.

Data management: A data extraction was prepared in Microsoft Excel sheet (Microsoft Corporation, Readmon, WA, USA) in accordance with the Cochrane Consumers and Communication Review Group's data extraction template (Group, 2016). The Excel sheet was used to assess inclusion requirements and subsequently tested for all selected studies. The process was independently conducted by the two authors (FMC and JA). Any disagreement regarding study eligibility was resolved in a discussion. Full text articles excluded, with reasons, were recorded. All the records were stored in the sheet.

Quality assessment / Risk of bias analysis: The version 2 of the Cochrane risk-of-bias tool for randomized trials (RoB2) (J. A. C.
Sterne et al., 2019) was used to assess the risk of bias in the included randomizedcontrolled trials. Five dimensions are inspected in this assessment tool: (i) bias arising from the randomization process; (ii) bias due to deviations from intended interventions; (iii) bias due to missing outcome data; (iv) bias in measurement of the outcome; and (v) bias in selection of the reported result. Using RoB2 a qualitative synthesis was performed. Two of the authors (JA and HS) independently assessed the risk of bias. Any disagreement in the rating was resolved through discussion and by a third author (FMC). The Cochrane risk of bias in nonrandomized studies of interventions (ROBINS-I) was used to assess the risk of bias in included non-randomized intervention studies (J. A. Sterne et al., 2016). Three domains are analyzed in this assessment tool: (i) pre-intervention (bias due to confounding; bias in selection of participants into the study); (ii) at intervention (bias in classification of interventions); and (iii) post-intervention (bias due to deviations from intended interventions; bias due to missing data; bias in measurement of outcomes; bias in selection of the reported results).

Strategy of data synthesis: The following information was extracted from the included original articles: (i) type of study design, number of participants ( $\mathrm{n}$ ), agegroup (youth, adults or both), sex (men, women or both), competitive level (if available), and type of original articles included (experimental, observational analytic); (ii) identification of the effects (acute and/or chronic), dimension of analysis (internal load [the measure of biological response to a given physical demand imposed by the exercise (Impellizzeri, Marcora, \& Coutts, 2019)] or biological responses in exercise; external load [the measure of physical demand or neuro-mechanical load imposed by the exercise (Impellizzeri et al., 2019)] or physical demands in exercise; recovery/ fatigue/readiness; psychological; fitness variations), outcomes explored, and main findings. 
Language: English.

Country(ies) involved: Portugal.

Keywords: Football; athletic performance; strength training; high-intensity interval training; resistance training.

Contributions of each author:

Author 1 - Jorge Ribeiro - Lead the project, ran the data search and methodological assessment, and wrote and revised the original manuscript.

Author 2 - Miguel Camões - Revised the original manuscript.

Author 3 - José Afonso - Ran the data search and methodological assessment and wrote and revised the original manuscript.

Author 4 - Hugo Sarmento - Executed the methodological assessment, wrote and revised the original manuscript.

Author 5 - Ricardo Lima - Revised the original manuscript.

Author 6 - Filipe Manuel Clemente - Lead the project, ran the data search and methodological assessment, and wrote and revised the original manuscript. 